\title{
Navigational User Interface Elements on the Left Side: Intuition of Designers or Experimental Evidence?
}

\author{
Andreas Holzinger ${ }^{1}$, Reinhold Scherer ${ }^{2}$, and Martina Ziefle ${ }^{3}$ \\ ${ }^{1}$ Medical University Graz, A-8036 Graz, Austria \\ Institute for Medical Informatics, Research Unit Human-Computer Interaction \\ Auenbruggerplatz 2/V, A-8036 Graz \\ andreas.holzinger@medunigraz . at \\ ${ }^{2}$ Graz University of Technology, Austria \\ Institute for Knowledge Discovery, Laboratory of Brain-Computer Interfaces \\ Krenngasse 37/IV, A-8010 Graz \\ reinhold.scherer@tugraz.at \\ ${ }^{3}$ RWTH Aachen University, Germany \\ Communication Science, Human Technology Centre (Humtec) \\ Theaterplatz 14, D-52062 Aachen \\ ziefle@humtec.rwth-aachen.de
}

\begin{abstract}
Humans tend to direct their attention toward the left half of their area of vision, which is known as visual pseudo neglect. Most navigational elements are placed at the left side. However, there is neither a theoretical reasoning nor empirical evidence, why these elements should be placed left. In the present study we examined three independent variables (presentation side of elements (left, right), number of elements (one, three, five) and a visual cue prior to selection (with cue, without). Dependent variables were selection times and accuracy of task completion. 50 participants were exposed to elements consisting of single words in bubbles. After clicking on the start element in the middle of the screen a number of elements were presented randomly on the left or right. In $50 \%$ of trials the presentation side was announced in advance, by using a visual cue. It was tested, whether and to what extent there is a preference and performance (correct selection time) increase for elements placed on the left side. When the cue was presented, performance increased; without cue information, elements on the left were selected faster. The use of cues resulted in no significant differences between the left and right side. A significantly better performance was found when only one element was presented on the left. With an increasing number of elements, the performance decreased. The results of this study suggest that the presentation of elements on the left side is advantageous for the speed of information processing only in the case of single elements. When selecting between numbers of options (three, five), placing elements on the left does not affect the selection performance.
\end{abstract}

Keywords: navigation, graphical user interface design, pseudo neglect, visual attention, performance, selection times. 


\section{Introduction and Motivation for Research}

It is common practice that user interface designers place navigational elements on the left side of user interfaces. Possibly, they are assuming an elementary benefit in information perception, encoding and cognitive processing.

Moreover, although there is only little empirical evidence (e.g. [1]), many design guidelines exist, which recommend to put navigational elements on the left side, e.g. [2], [3], [4], . However, an underlying theory along with an experimental proof seems not to exist. The "rule to arrange navigational buttons on the left" [5] seems to be a well-known designer intuition, not grounded in theory. However, we must be careful with the term "intuitive", which is not properly defined; any common definition of the term makes its use inappropriate in interface design [6].

Interestingly, we experienced that when designers placed navigational elements explicitly not on the left side, e.g. at the right side (as in figure 1), end users expressed that they "intuitively" did not like it - possibly because they are used to have the navigational elements on the right side. Motivated by these practical experiences, we began to think for a possible reason, a theory and for an experimental setting to answer the question.

Consequently, this study aims at a critical investigation, whether and to what extent the positioning of navigational elements does factually benefit selection performance. In section 2 we provide some related work on which we build our work, in section 3 we provide some necessary theoretical background, in section 4 we present our questions addressed and the research logic of our experiment, in section 5 we describe our methods and materials used, in section 6 we present our results and in section 7 we draw conclusions and provide some future research outlook.

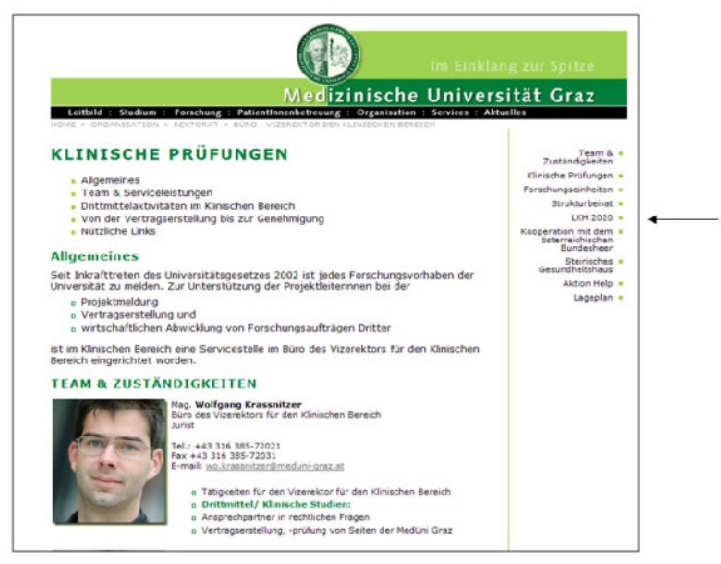

Fig. 1. After the „modern“ designers placed the navigational elements on the right side, end users massively complained that they "do intuitively feel that this is wrong"

\section{Related Work}

Kalbach \& Bosenick (2003) [1] compared two Web page layouts (see figure 2): one with the main site navigation menu on the left of the page, and one with the main site 
navigation menu on the right. $\mathrm{N}=64$ participants (44 of them female) were divided equally into two groups and assigned to either the left-hand or the right-hand navigation test condition. Using a stopwatch, the time to complete each of five tasks was measured. The hypothesis that the left-hand navigation would perform significantly faster than the right-hand navigation was not supported. Instead, there was no significant difference in completion times between the two test conditions. However, this work questioned the leading Web design thought as well as the common practice, that the main navigation menu should always be left justified. In their experiments, the participants were asked to carry out 6 different tasks, which required the use of the navigational elements. Interestingly, in average, the time to perform the tasks was about 3.7 seconds shorter when elements were placed on the left side (left $\mathrm{M}=30.5 \mathrm{SD}=34.3$; right $\mathrm{M}=34.2 \mathrm{SD}=35.8$ ). Kalbach \& Bosenick performed an ANOVA for all tasks, however, again there were no significant difference between both conditions.

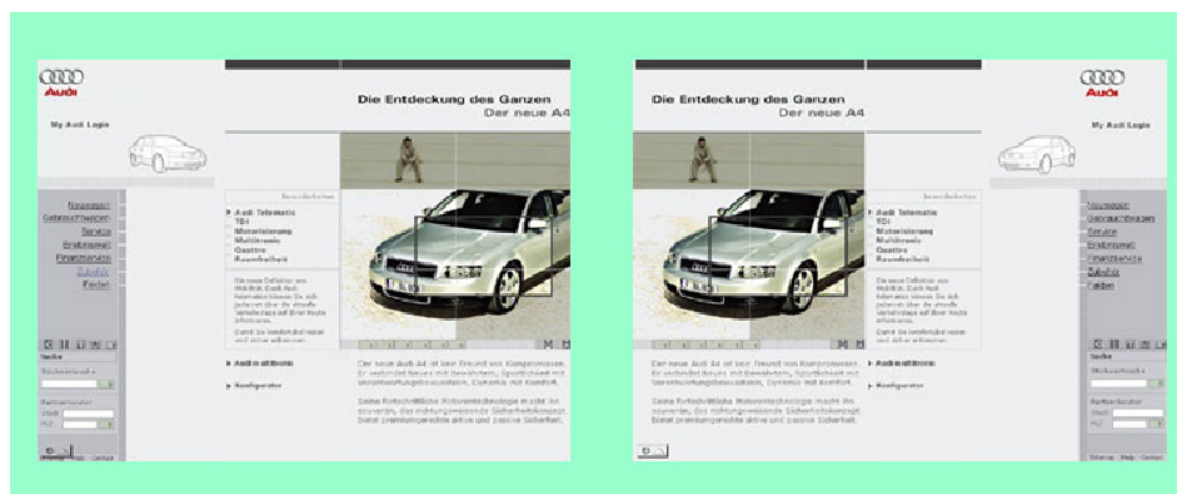

Fig. 2. The tested Webpages of the experiment done by Kalbach \& Bosenick [1]

Van Schaik \& Ling (2001) [7] empirically evaluated design guidelines according to the arrangement of navigational elements. They wanted to discover possible effects of the frame layout (the arrangement of the navigational elements) along with the contrast of the background on visual search performance. There were two independent variables (background - same and different contrast) and the frame layout (arrangement of the navigational elements: left, right, top, down). As experimental material they used a Webpage, which consisted of two parts: main frame layout and menu frame layout. The latter contained five hypertext links in blue text colour and a word length of 5 to 10 letters. They presented the navigational elements on different areas of the page (left, right, top, down) and measured the accuracy and the time of right and wrong answers and the subjective preference for a certain design. In their study they observed $\mathrm{N}=189$ participants (146 of them female) and allocated 90 participants to the experimental condition "same contrast" and 99 participants to "different contrast". In the first experimental condition the navigational frame layout and the main frame layout had both a grey background, in the other condition the navigational frame was white and the main had grey colour. Within the experiment they presented at first a blank white screen and then a word (target, black colour). 
Afterwards, the participants had to find this word out of the five hypertext links. The word was either presented or hidden. Only if the participants found the word they pressed a button.

Van Schaik and Ling analysed the hits and the misses and measured reaction times. A main effect was detected amongst the hits $[\mathrm{F}(3,561)=4,473, \mathrm{p}<0,01]$, on the right side $(M=97,47, S D=6,84)$ there were less hits as on top $(M=98,88, S D=5,79)$ or on the left side $(\mathrm{M}=98,77, \mathrm{SD}=5,17)$ (Tukey's Honestly Significant Difference), $\mathrm{p}<0.05$ ). The reaction time (in milliseconds) revealed also a main effect $[\mathrm{F}(3,561)=39,910$, $\mathrm{p}<0,001]$; right $(\mathrm{M}=1371, \mathrm{SD}=269)$ and down much longer times were measured than on top ( $\mathrm{M}=1302, \mathrm{SD}=300)$ or left $(\mathrm{M}=1305, \mathrm{SD}=235)$ (Tukey's HSD, $\mathrm{p}<0,05)$.

In conclusion the authors recommend arranging navigational elements on the left side or on top and they tried to explain these effects on intercultural influences (e.g. reading culture).

\section{Theoretical Background}

\subsection{Visual Pseudoneglect}

It is known that humans primarily attend to objects on the left side of space as could be shown in classic cancellation tasks routinely used during neuropsychological testing (see figure 3, second from the left, and compare figure 4).
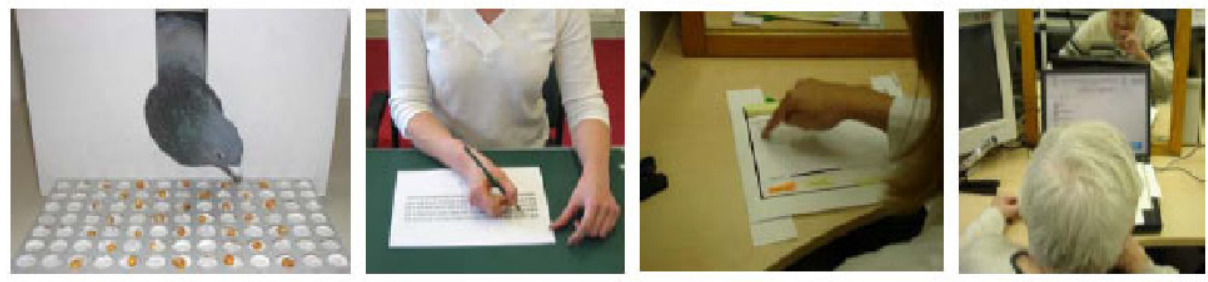

Fig. 3. Left (1-2): Settings to test effects on visual pseudoneglect [8]; Right (3-4): Settings where such effects were observed with humans [9]

Neglect and pseudoneglect are asymmetries of spatial attention, which are often assumed to have fundamental theoretical and neurological relationship to each other, although there is much dispute about it.

Basically, when neurologically normal individuals bisect a horizontal line as accurately as possible, they reliably show a slight leftward error. This leftward inaccuracy is known as pseudoneglect [10], [11], [12].

Diekamp et al. (2005) [8] (see figure 3 (left) and figure 4) tested birds in a task that closely matches these cancellation tasks: the birds were required to explore an area in front of them and to sample grains. The results showed that birds displayed a clear bias into the left side, as evident in the pecking activity or the order in which pecks were placed in the left or right side. Let's go back to humans: Line bisection tasks are a commonly used procedure for testing spatial attention. 
Several studies before have found that patients with a so called left hemisphereneglect, bisect long lines too far to the right, but bisect short lines too far to the left, and some studies reported that normal participants bisect long lines too far to the left, presumably reflecting an over-estimation of the left side due to the role of the right hemisphere in attention [13]. Jewell \& McCourt (2000) [11] provide a large-scale review of the literature concerning visual and non-visual line bisection in 2191 neurologically normal subjects. The meta-analytic results indicate a significant leftward bisection error in neurologically normal subjects, with an overall effect size of between -0.37 and -0.44 (depending on integration method).

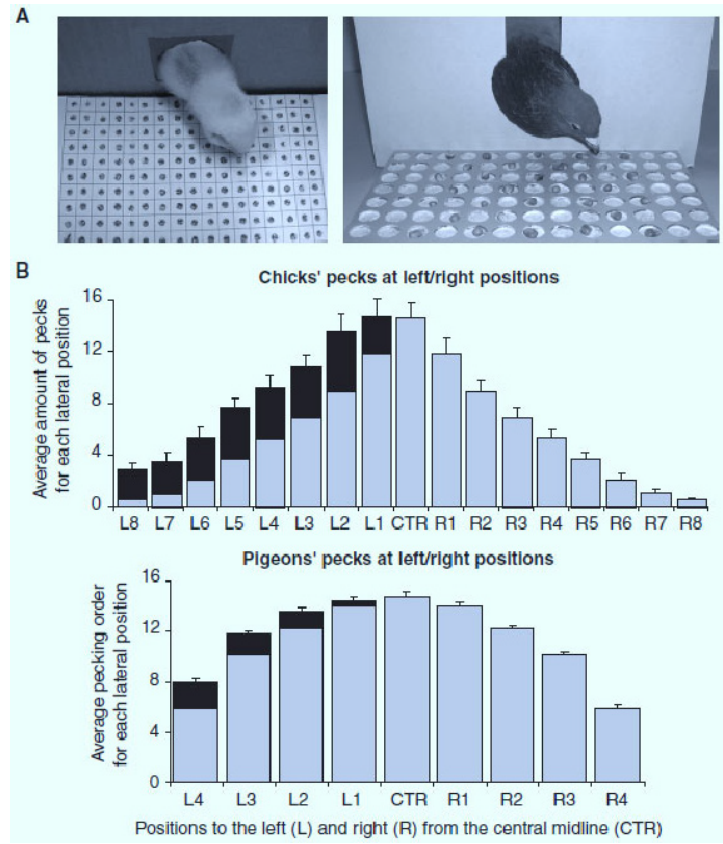

Fig. 4. The results from Diekamp et al. (2005) [8]

\subsection{Fitt's Law and Hick's Law: Human Performance Principles}

No paper about navigation can ignore to mention these two laws. According to Fitts (1954), [14] the average duration of responses is directly proportional to the minimum average amount of information per response.

Fitts also discovered that movement time is a logarithmic function of distance when target size was held constant, and that movement time is also a logarithmic function of target size when distance was held constant; the time to acquire a target is therefore a function of the distance to and size of the target. Fitts' law has been applied to user interface design [15]. The time for pointing tp is a function of both the distance $d$ of the reach and the width $w$ of the target object. This relationship can be expressed in the following formula where $a$ and $b$ are empirical constants and vary depending the used device: 


$$
\text { tp }=a+b * \log 2(2 d / w)
$$

The expression $\log 2(2 \mathrm{~d} / \mathrm{w})$ is, according to Shneiderman (1997), [16] also called index of difficulty (ID). Understanding how manipulations of $d$ and $w$ affect the time $t p$ is of considerable importance in developing efficient user interfaces. For our study, we had, within certain parameters (including the dimensions of the screen), full control over the size of target objects and the distance of the user from the screen, and we had experience from previous work [9], [17].

However, we must emphasize, that the formula $a+b * \log 2(2 d / w)$ is not quite correct. Consider the case where e.g. your finger happens to be where you wish to touch, when a new screen comes up. In that case $d=0$, and $\log (0)$ is undefined, so the formula provides no result (it should give a as the time).

Similar to Fitt's Law, the so called Hick's Law [18], describes the time it takes to make a decision as a result of the possible choices of the end user; consequently it assesses cognitive information capacity in choice reaction experiments. The reaction time (rt) raises logarithmically, if the amount of alternatives raise:

$$
\mathrm{rt}=\mathrm{k} * \log 2(\mathrm{n}+1)
$$

Both laws are two surviving human performance principles based originally on Shannon and Weaver's (1949) Information Theory concept [19]. With the advent of user interfaces, these laws were used as the fundamental design principles [20]. A good discussion on the impact of those two laws can be found in [21].

\section{Questions Addressed and Research Logic of the Experiment}

We conducted an experiment in which participants were instructed to select a target element, which was either placed on the left or the right side of the display. In addition, we examined if the directing of visual attention (using a cue) facilitates selection performance differentially for elements placed on the left and right side. Also we varied the number of distracting elements (the target stimulus had to be selected as a singleton as well as among three or five elements) and determined whether the number of elements asymmetrically influences the processing of information on the left and right side of the display.

\section{Methods}

\subsection{Independent and Dependent Variables}

Three independent variables were examined:

(1) The first independent variable is the side on which the target is displayed, comparing elements on the left and the right side of the display.

(2) The second independent variable is the presentation of a visual cue (directing visual attention), directly before the target had to be selected (both with cue, and without cue). 
(3) The third independent variable is the number of elements, which were displayed on either side of the screen, differentiating one, three or five elements. The cuing as independent variable in combination with the side on which navigational elements are presented allows us to understand whether the selection performance on both sides is equally high under the cueing condition (if visual attention is beneficial then we should not find the left side benefit). In the condition without cuing we would expect the left side benefit. The arrow (the cue) did not disappear in the cuing conditions, but was not present in the non-cuing conditions (see figure 5).

Dependent variables included the speed and accuracy of performance. For the speed, the selection times (in ms) were determined (executed by a computer mouse). Regarding accuracy, the percentage of correct answers was counted.

\subsection{Hypotheses}

The following hypotheses were underlying the experiment:

I. A significant main effect for the side at which elements were displayed

- Faster processing times for elements placed on the left side of the display compared to elements placed on the right side of the display

- A higher accuracy of answers for elements placed on the left side of the display compared to elements placed on the right side of the display

II. A significant main effect for directing visual attention

- Cuing the position of the expected elements prior to displaying it increases performance in terms of speed and accuracy of selection times, independently from the side at which elements are displayed.

III. A significant main effect for the number of elements, among which the target stimulus has to be identified

- The number of elements displayed on either side (right or left) decreases performance in terms of speed and accuracy of selection times

\subsection{Experimental Task}

The task of each participant was to select a target word, which was positioned either on the left or the right side of the display. They started each trial by clicking on a centrally positioned element (word). In $50 \%$ of the trials the central element contained a visual cue (a bold arrow) above the start element, which indicated the presentation side of the target element. After participants clicked on the start element it disappeared and the target element(s) - one, three or five elements - appeared. The trial was finished when participants had clicked on the correct target. In order to meet requirements of ecological validity, no time restrictions were given. Participants were instructed to execute the trails in a normal easy and efficient working speed. In Figure 5 , a screenshot of the cue (a bold arrow above the start element) is depicted which indicates the side at which the navigational elements would appear.

After selecting, the start element (a word) disappeared and the target element (the same word) on the left or right side appeared and had to be selected. Figure 6 shows the display for a trial without a visual cue, and three elements on the left side (the central element is the target word). The distance from the start element to the 


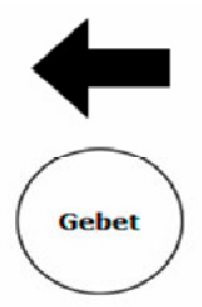

Fig. 5. Start element with a cue for the positioning of displaying side of the target element

first/third element (3-element condition) was $11.5 \mathrm{~cm}$ and to the second/forth element (5 element-condition) $12.5 \mathrm{~cm}$. The position of the target word was randomized across trials.

All words were between 4 to 7 letters and represented highly familiar words within the German language. The different word lengths were chosen to meet requirements of ecological validity: Normally, we process different word lengths when using websites and selecting objects. To simulate such a real-life scenario we also presented different word lengths in the study. As all experimental stimuli represented highly frequent words, we can exclude confounding effects of different word frequencies on selection times (which are well known). As all participants received the same stimulus material, word length did not asymmetrically influence the processing across participants. In order to learn if the word length has an impact on reaction times we analyzed this factor. No significant effect of word length was found, therefore we can exclude confounding effects of different word lengths.

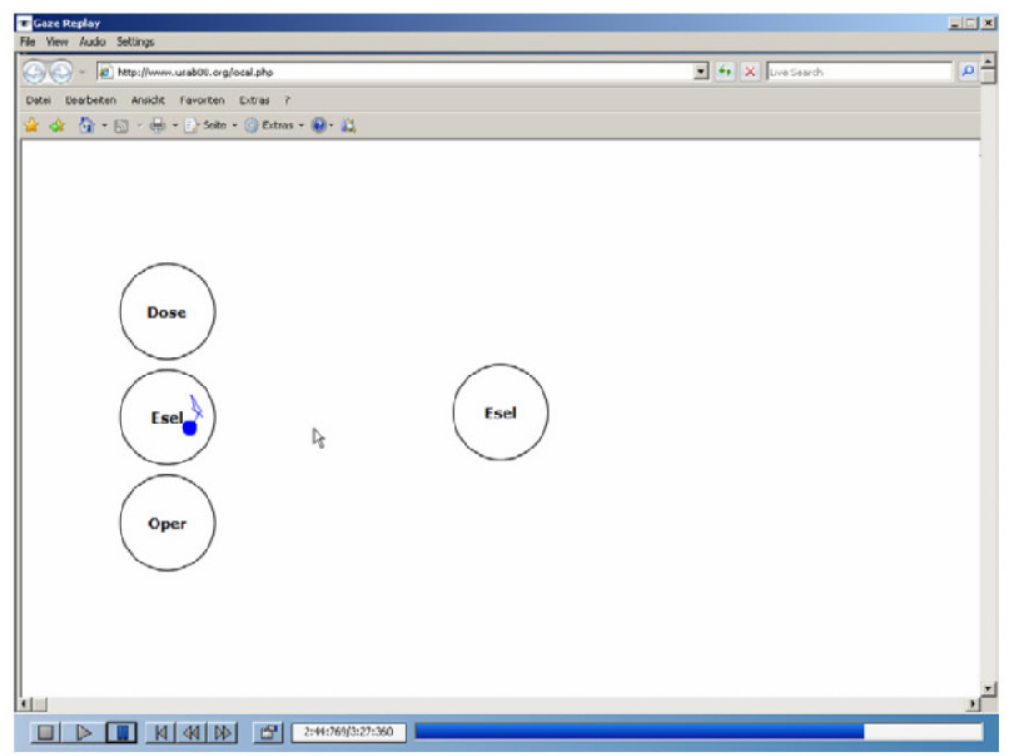

Fig. 6. Screenshot for a trial without a visual cue, and three elements on the left side (the central element is the target word) 
The distance from the center of the start element to the center of the target element (on the left or right side) was $11 \mathrm{~cm}$ and 10.38 degree (visual angle).

\subsection{Participants}

A total of $\mathrm{N}=50$ participants volunteered to take part in the experiment. Simulating the diversity of computer users, the age range varied (between 10 and 64 years of age, $\mathrm{M}=32.4 ; \mathrm{SD}=11.8$ ) as well as the educational level. 16 participants had a graduation from apprenticeship training, 4 persons had a vocational school degree; 22 Participants reported to have a general qualification for university entrance and 8 persons had completed a university diploma.

Participants were surveyed regarding their experience with computers and Internet usage as well as their self-reported computer literacy. All persons reported to be frequent computer users with a satisfying experience when using computers and Internet. 35 persons indicated to use the Internet about 5-7 times per week, 9 persons reported to use the Internet about 1-2 times a week. 6 persons - the older ones reported to use the Computer and the Internet to a lesser extent (about once a month). All persons had a normal visual acuity. 26 out of 50 persons wore their corrective lenses during the experiment. Participants were instructed to use the computer mouse with their dominant hand (all participants were right handed), as tested by a handdominance testing procedure. Participation was voluntarily and the participants were not gratified for their efforts.

\subsection{Apparatus and Materials}

For the equipment, a standard working place was used. Participants sat in front of a computer screen, using a computer mouse (optical Logitech Wheel Mouse). In the beginning of the experiment, participants adjusted the height of the chair according to individual needs. In order to exclude different body movements and sighting conditions across participants, a chin rest was used which did not restrict participants, but allowed to control for comparable viewing conditions (figure 7).

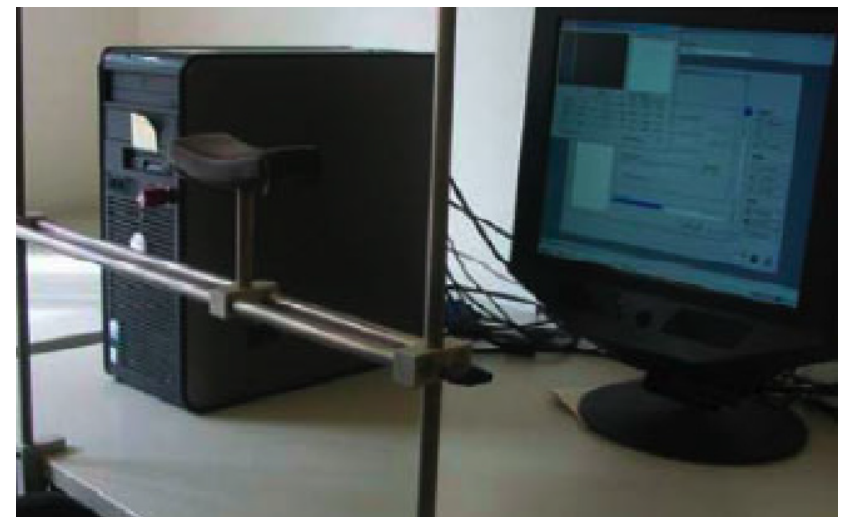

Fig. 7. The Experimental setting including a Tobii 1750 eye tracker with chin rest 
The stimulus material consisted of disyllabic (german) words (4-7 letters). The selected words are common in the everyday German language usage; thus we can exclude confounding effects from word processing of difficult or unfamiliar material. Moreover, the stimuli were presented in positive polarity (black letters on a white background).

\subsection{Experimental Design and Procedure}

The experiment was based on a 3 (number of elements) x 2 (presentation side of navigational elements) $\times 2$ (with and without cue) - design for repeated measurements. All participants absolved all conditions.

The navigation side at which stimuli were located (right or left) and the number of elements (one, three or five) was varied randomly across trials. The cuing variable was blocked, with one half of participants starting with the order "with cue, followed by without cue" the other half vice versa ("without cue, followed by with cue"). As checked by ANOVA procedures, the order did not impact visual performance.

Overall, 108 trials had to be carried out (54 trials with and 54 trials without a cue, 27 on the left and 27 trials on the right side). The whole experiment lasted about 3040 minutes, depending on the individual working speed.

In order to meet the high control demands in experiments, we did not use a real webpage, but used a simulation.

\section{Results}

Data were analyzed by analyses of variance for repeated measurements assessing effects of (1) the presentation side, (2) the presence/absence of a visual cue and (3), the number of elements presented on the interface at a time.

The significance of the F-Tests was taken from Pillai values. The level of significance was set at $\mathrm{p}<0.05$. First, the main effects are reported, followed by interacting effects. The data revealed an accuracy level of 100\%: None of participants had difficulties executing the selection of elements and perfectly carried out the tasks. Therefore, in the following, the selection times are focused at.

\subsection{Main Effect of Presentation Side}

Participants needed, on average, $1155.7 \mathrm{~ms}(\mathrm{SD}=223.6 \mathrm{~ms}, \mathrm{SE}=30.7 \mathrm{~ms})$ for the selection of the target elements presented on the left side compared to $1181.5 \mathrm{~ms}$ (SD $=244 \mathrm{~ms}, \mathrm{SE}=33.2 \mathrm{~ms}$ ) for the selection of elements, which are placed on the right side of the display.

The small mean difference of $26 \mathrm{~ms}$ revealed to be statistically significant (Figure 8 left) yielding a meaningful main effect of presentation side $(\mathrm{F}(1,49)=11.8$; $\mathrm{p}<$ $0.05)$.

\subsection{Main Effect of Cuing Visual Attention}

Cuing visual attention facilitates the information processing: Conditions, in which a visual cue announced the presentation side of the elements, were significantly faster 

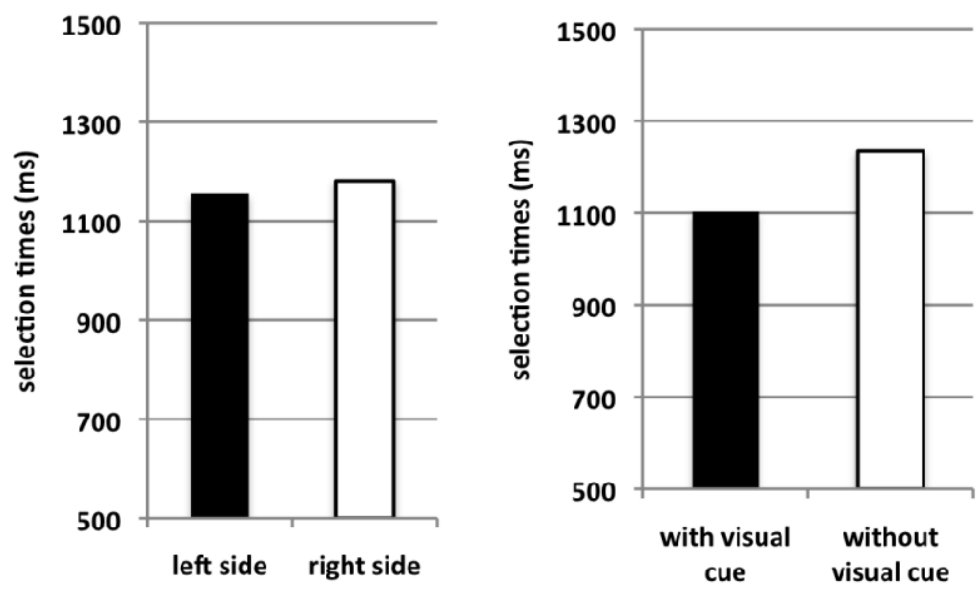

Fig. 8. Left: mean selection time in ms for the left compared to the right presentation side of elements; right: mean selection times in ms for trials with and without visual cue

processed $(\mathrm{M}=1102.6 \mathrm{~ms} ; \mathrm{SD}=240.3 \mathrm{~ms}, \mathrm{SE}=32.22 \mathrm{~ms})$ compared to trials without visual cuing $(\mathrm{M}=1234.6 \mathrm{~ms} ; \mathrm{SD}=249.1 \mathrm{~ms}, \mathrm{SE}=32.74 \mathrm{~ms})$.

The mean benefit of $132 \mathrm{~ms}$ represented another significant main effect $(\mathrm{F}(1,49)=$ 89.3; $\mathrm{p}<0.05$ ). Outcomes are visualized in Figure 8 (right side).

\subsection{Main Effect of the Number of Elements Presented at a Time on the Screen}

Furthermore, the number of elements among which the target element had to be selected also showed a significant impact on the identification and selection of navigational elements $(\mathrm{F}(2,48)=378.4 ; \mathrm{p}<0.05))$. The outcomes are visualized in figure 9.

Descriptive outcomes show that selection times were considerably faster for conditions with only one element $(\mathrm{M}=978.9 \mathrm{~ms} ; \mathrm{SE}=31.4 \mathrm{~ms})$, compared to three elements $(\mathrm{M}=1140.2 \mathrm{~ms} ; \mathrm{SE}=31.9 \mathrm{~ms})$ and five elements $(\mathrm{M}=1386.7 \mathrm{~ms} ; \mathrm{SE}=$ $34.4 \mathrm{~ms})$.

The interacting effects between the three main factors are of considerable interest, as it shows whether the (small) advantage of the left side presentation of navigational element also holds when the identification and selection of target elements is facilitated by cuing the visual attention and when the number of elements which have to be processed at the same time increases.

On a first sight, we expect that cuing of visual attention reduces the left side benefit and that the left side advantage profits from a small number of elements to be processed at a time. Outcomes of the interacting effects are reported in the next sections.

\subsection{Interacting Effect of Visual Cueing and Presentation Side}

A significant interaction effect was found for the visual cuing of attention and the side on which navigational elements were positioned $(\mathrm{F}(1,49)=11.3 ; \mathrm{p}<0.05$, see figure 10$)$. 


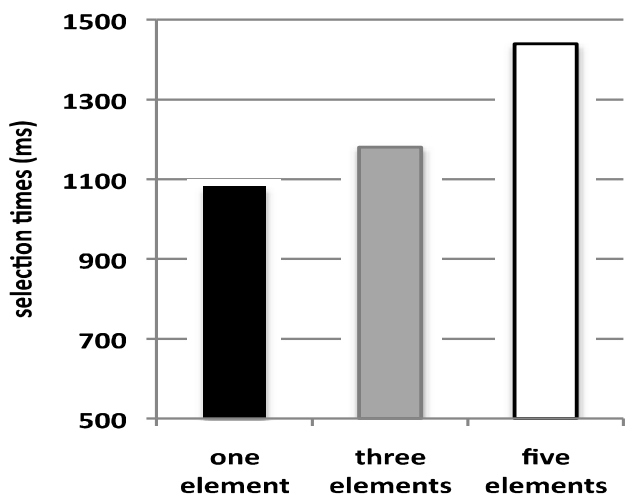

Fig. 9. Mean selection time in ms for one, three and five elements presented on either side of the display

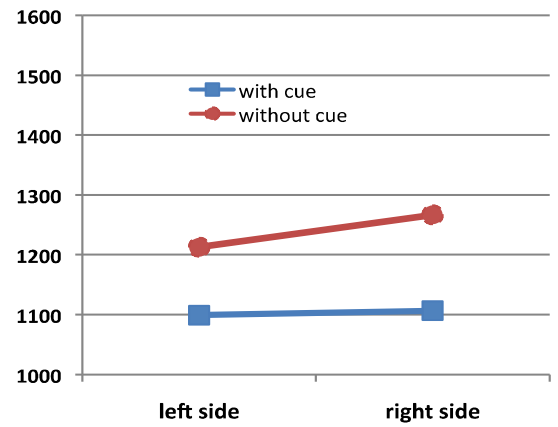

Fig. 10. Interacting effect of visual cue and presentation side (mean selection times in ms)

As can be seen from figure 10, the detrimental effect of having no visual cue that indicates the side of presentation is asymmetrical for the left and the right side: the selection times are slowest in conditions in which no visual cueing is present and the elements that have to be processed are on the right side. In other words this can be referred to as an indirectly evidence for the benefit of the left-side presentation as elements on the left side are disadvantaged to a lesser extent when there is no cuing of visual attention.

\subsection{Interaction Effect of Visual Cueing and Number of Elements}

In this analysis we examined whether the cuing of visual attention is equally beneficial for the number of navigational elements that are presented at a time. Analyses revealed a significant effect $(\mathrm{F}(2,48)=13.7 ; \mathrm{p}<0.05$, figure 11): Performance drastically varied depending on the number of elements and a visual cue. 


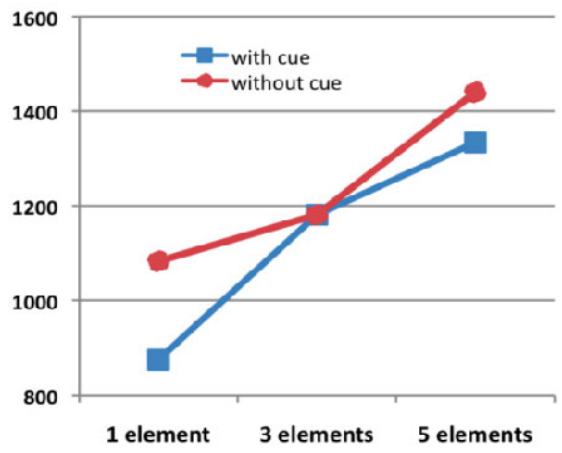

Fig. 11. Interacting effect of visual cue and presentation side (mean selection times in ms)

The best performance was given, when a visual cue indicated the presentation of one element only ( $\mathrm{M}=875 \mathrm{~ms} ; \mathrm{SE}=28.73 \mathrm{~ms})$, the worst performance was found in the condition in which no visual cue was given and five elements had to be processed, among which the target element had to be selected $(M=1439.9 \mathrm{~ms} ; \mathrm{SE}=27.73 \mathrm{~ms})$. Visual cuing facilitates the processing of elements and reduces the detrimental effects of having more than one element.

\subsection{Interacting Effect of Presentation Side and Number of Elements}

Finally it was analyzed if there is a significant interaction of presentation side and the number of elements among which the target element had to be selected. As can be seen from figure 12 , there is a significant effect $(\mathrm{F}(1,49)=11.3 ; \mathrm{p}<0.05)$.

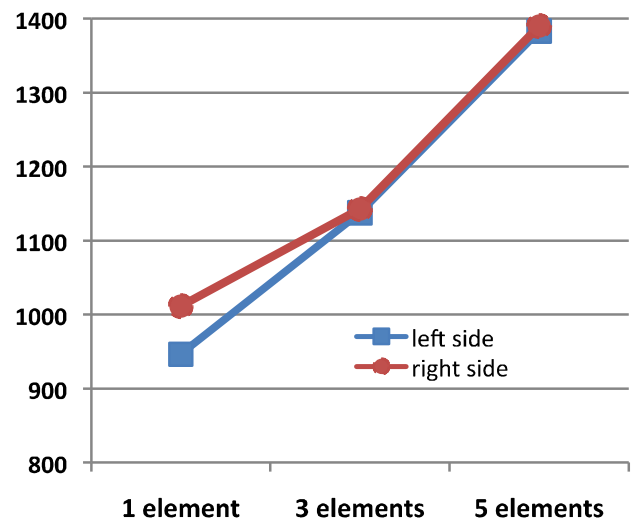

Fig. 12. Interacting effect presentation side and number of elements that have to be processed at a time (mean selection times in $\mathrm{ms}$ ) 
From an ergonomic point of view, this interacting effect is most insightful as it shows that the benefit of the left-side presentation of elements is only valid when one element is depicted (what is rather unusual in real web sites). Whenever the number of navigational elements increases (three, five elements) the benefit vanishes and shows the very same selection times.

\section{Conclusion and Future Research Outlook}

The results of the presented center-out selection study show that placing elements on the left side significantly decreases the selection time only in cases were individuals elements have to be selected. When user had to choose between several options, no significant differences between left or right were found. As expected, the use of cues decreases the overall selection time. The availability of the a-priori information on the position, however, eliminates also the enhanced left-sided performance for individual elements. The number of elements was of no direct influence on the performance time. There has been an increase in time to perform task with one, three and five elements.

As described in Hick's law, there is a correlation between the number of reaction alternatives and the reaction time. The more complex one task, the longer one needs for the task. In terms of cognitive performance there were no differences between both sides. This is an important finding as this suggests that the performance of usery that are engaged in a selection task, such as navigating a webpage with several menu elements, is not affected by the side the elements are shown on the screen. Phenomena like the pseudoneglect or the intrinsic turning behavior in right-handers, i.e., turning toward the left side due to hemispheric dopamine asymmetries [22], may argue against such a behavior.

Possible explanations for the enhanced performance during the perception of individual stimuli on the left side could be the biomechanics of the hand and the influence of the writing culture. As known there are many differences between western and eastern cultures, which have a direct influence on designing user interfaces.

This brings us directly to the limitations of this study, consequently our further research duties: This study was conducted only with German speaking participants; hence the results cannot be generalized to other cultural areas - especially in terms of reading habits. It would be very interesting to conduct a similar study in a language with a completely different orthography (e.g. Japanese). By this, we could learn, whether and to what extend the effects are biased by alphabetical writing systems. Also, as the reading direction could also impact the left-side benefit, it would be insightful to replicate the study in a language with a reverse reading direction (as e.g Hebraic or Arabian language).

A further limitation is that we used a webpage simulation in a laboratory experiment, and thus in real-life there could be a lot of different influences with different additional effects. For example, due to experimental requirements the distance between user and interface was fixed and controlled. In contrast, in real using settings, end users do naturally move in front of the screen, resulting in different distance and angles. 
In future work different target elements should be used, e.g. symbols. Currently we have developed an online experimental tool, were we plan to replicate this studies within an intercultural context on a large scale.

Summarizing, the best performance was achieved when selecting individual elements on the left side of the screen. Besides from this, when end users have no cue or several options to choose from, the side does not significantly impact on the performance. However, due to the fact that the majority of the population is righthanded, and right-handers tend to focus on the left side, placing navigational elements on the left side may be the most intuitive position. Besides this, end users are accustomed to it and they expect it there; consequently the first designers were intuitively right, and we can conclude, yes, it was proper intuition - and it is not recommendable for "modern designers" to switch to the other side.

Acknowledgements. We are grateful for the valuable comments of the anonymous reviewers. Many thanks to the students Sylvia Graf and Stefan Mayr for their work on programming and experimenting, and to Prof. Dietrich Albert for his support of this work.

\section{References}

1. Kalbach, J., Bosenick, T.: Web page layout: A comparison between left and right-justified navigation menues. Online Journal of Digital Information (2011), http://journals.tdl.org/jodi/article/view/94/93

(last access: January 10, 2011)

2. Koyani, S.J., Bailey, R.W., Nall, J.R.: Research-Based Web Design \& Usability Guidelines (2010),

http://usability.gov/pdfs/guidelines_book.pdf (last access: December 20, 2010)

3. Torres, R.J.: User Interface Design and Development. Prentice Hall, Upper Saddle River (2002)

4. Preece, J., Sharp, H., Rogers, Y.: Interaction Design: Beyond Human-Computer Interaction. Wiley, New York (2002)

5. Johnson, J.: GUI Bloopers 2. 0: Common User Interface Design Don'ts and DOS. Morgan Kaufmann Series in Interactive Technologies. Morgan Kaufmann, Amsterdam (2007)

6. Raskin, J.: Intuitive = Familiar. Communications of the ACM 37(9), 17 (1994)

7. van Schaik, P., Ling, J.: The effects of frame layout and differential background contrast on visual search performance in web pages. Interacting with Computers 13, 513-525 (2001)

8. Diekamp, B., Regolin, L., Güntürkün, O., Vallortigara, G.: A left-sided visuospatial bias in birds. Current Biology 15(10), 372-373 (2005)

9. Holzinger, A.: Finger Instead of Mouse: Touch Screens as a Means of Enhancing Universal Access. In: Carbonell, N., Stephanidis, C. (eds.) UI4ALL 2002. LNCS, vol. 2615, pp. 387-397. Springer, Heidelberg (2003)

10. Porac, C., Searleman, A., Karagiannakis, K.: Pseudoneglect: Evidence for both perceptual and attentional factors. Brain and Cognition 61(3), 305-311 (2006)

11. Jewell, G., McCourt, M.E.: Pseudoneglect: A review and meta-analysis of performance factors in line bisection tasks. Neuropsychologia 38(1), 93-110 (2000) 
12. McCourt, M.E., Jewell, G.: Visuospatial attention in line bisection: stimulusmodulation of pseudoneglect. Neuropsychologia 37(7), 843-855 (1999)

13. Rueckert, L., Deravanesian, A., Baboorian, D., Lacalamita, A., Repplinger, M.: Pseudoneglect and the cross-over effect. Neuropsychologia 40(2), 162 (2002)

14. Fitts, P.M.: The Information Capacity of the Human Motor System in Controlling the Amplitude of Movement. Journal of Experimental Psychology 47(6), 381-391 (1954)

15. MacKenzie, I.S., Tatu, K., Miika, S.: Accuracy measures for evaluating computer pointing devices. In: Proceedings of the SIGCHI Conference on Human Factors in Computing Systems, pp. 9-16 (2001)

16. Shneiderman, B.: Designing the User Interface. In: Strategies for Effective HumanComputer Interaction, 3rd edn., Addison-Wesley, Reading (1997)

17. Holzinger, A., Höller, M., Schedlbauer, M., Urlesberger, B.: An Investigation of Finger versus Stylus Input in Medical Scenarios. In: Luzar-Stiffler, V., Dobric, V.H., Bekic, Z. (eds.) ITI 2008: 30th International Conference on Information Technology Interfaces, pp. 433-438. IEEE, Los Alamitos (2008)

18. Hick, W.E.: On the rate of gain of information. Quarterly Journal of Experimental Psychology 4, 11-26 (1952)

19. Shannon, C.E., Weaver, W.: The Mathematical Theory of Communication. University of Illinois Press, Urbana (1949)

20. Card, S.K., Moran, T.P., Newell, A.: The psychology of Human-Computer Interaction. Erlbaum, Hillsdale (1983)

21. Seow, S.C.: Information theoretic models of HCI: A comparison of the Hick-Hyman law and Fitts' Law. In: Human-Computer Interaction, vol. 20(3), pp. 315-352 (2005)

22. Mohr, C., Landis, T., Bracha, H.S., Brugger, P.: Opposite turning behavior in righthanders and non-right-handers suggests a link between handedness and cerebral dopamine asymmetries. Behavioral Neuroscience 117(6), 1448-1452 (2003) 